\title{
On the Evolutionary Defense of Scientific Antirealism
}

\begin{abstract}
Van Fraassen (1980) claims that successful theories exist today because successful theories survive and unsuccessful ones die. Wray $(2007,2010)$ appeals to Stanford's new pessimistic induction (2006), arguing that van Fraassen's selectionist explanation is better than the realist explanation that successful theories exist because they are approximately true. I argue that if the pessimistic induction is correct, then the evolutionary explanation is neither true nor empirically adequate, and that realism is better than selectionism because realism explains more phenomena in science than selectionism.
\end{abstract}

\section{Keywords}

Evolutionary Theory, Pessimistic Induction, Scientific Realism, Success of Science

Park, Seungbae (2014). "On the Evolutionary Defense of Scientific Antirealism”, Axiomathes 24 (2): 263-273.

Seungbae Park

Division of General Studies

Ulsan National Institute of Science and Technology

The Republic of Korea

nature@unist.ac.kr

\section{Introduction}

Science is successful. We can produce new crops and animals by manipulating genes. We can grow human organs by using stem cells. We can see celestial bodies billions of light years away from the earth. We can monitor asteroids in the solar system and predict collisions with the earth. Science liberated us from the fear of unexplainable phenomena, such as solar and lunar eclipses. Why is science so successful? What can explain the success of science?

Putnam (1975) and van Fraassen (1980) provide different explanations of the success of science. However, the phrase 'the success of science' is ambiguous. Although the realist and the antirealist aim to explain the success of science, they ultimately explain different phenomena of science due to the ambiguity of the phrase. In this paper, I will clarify the different meanings of 'the success of science' before critically examining the evolutionary explanation and demonstrating the superiority of the realist explanation to the evolutionary explanation.

Wray $(2007,2010)$ contends that the evolutionary explanation is superior to the realist explanation, appealing to evolutionary theory and Stanford's new pessimistic induction (2006). I object that it is self-undermining for the antirealist to use evolutionary theory to explain the success of science, and self-defeating for the antirealist to employ the pessimistic induction to demonstrate the superiority of the evolutionary explanation over the realist explanation. I also argue that the realist explanation has a broader scope than the evolutionary explanation. Finally, I vindicate the realist predicate 'approximate truth.'

\section{Putnam vs. van Fraassen}

Putnam (1975) argues that science is successful because some scientific theories are approximately true. A theory is successful when it "has led to confirmed predictions and has been of broad explanatory scope" (Laudan, 1981: 23). Thus, Putnam's suggestion is that theories have the capabilities to explain and predict phenomena as well as to manipulate 
things because they approximately reflect unobservables. If DNA or something like DNA does not exist, we would not be able to produce new crops and animals. In short, it would be a miracle if successful theories are not even approximately true.

Like Putnam, van Fraassen claims that the success of science cries out for an explanation. We need to explain why current scientific theories regularly make true predictions:

Science, apparently, is required to explain its own success. There is this regularity in the world, that scientific predictions are regularly fulfilled; and this regularity, too, needs an explanation. (van Fraassen, 1980: 39)

Van Fraassen offers an evolutionary story of why science is successful. He claims that current science regularly makes true predictions because successful theories survive and unsuccessful ones die, similarly to biological organisms:

..I claim that the success of current scientific theories is no miracle. It is not even surprising to the scientific (Darwinist) mind. For any scientific theory is born into a life of fierce competition, a jungle red in tooth and claw. Only the successful theories survive - the ones which in fact latched on to actual regularities in nature. (van Fraassen, 1980: 40)

The success of science can be explained by the selection process in which successful theories are selected and unsuccessful theories are eliminated. Van Fraassen's position might be called selectionism in Wray's terminology $(2007,2010)$.

However, the selectionist explanation is somewhat elusive. We first need to clarify what selectionism aims to explain. Its explanandum is either the fact that we have successful theories or the fact that a particular theory is successful. In other words, the evolutionary explanation is an answer either to the question "Why do we have successful theories?" or to the question "Why is a particular theory successful?" There is a huge difference between these two questions, as Wray (2007: 83) aptly notes.

Let me explicate the difference with examples. Consider the many successful theories in contemporary science, such as the kinetic theory of heat, the oxygen theory of combustion, the special theory of relativity, evolutionary theory, and cell theory. Why do we have such theories? Why do successful theories exist today? This question seeks information regarding the historical process that produced the successful theories. In contrast, consider that the kinetic theory of heat can explain and predict heat phenomena. Why is it successful? This question seeks information regarding a semantic property of the kinetic theory of heat that enables the theory's success.

We must be clear about what philosophers are in fact explaining when they explain the success of science. It is clear that Putnam's explanandum is the fact that a theory is successful. Van Fraassen's explanandum is either the fact that a theory is successful or the fact that we have successful theories. In the following sections, I will explore the problematic consequences under each interpretation.

\section{Why Is a Theory Successful?}

Suppose that the explanandum of the evolutionary explanation is the fact that a theory is successful. Then, van Fraassen's proposal is that a theory is successful because successful theories survive and unsuccessful ones die. An immediate objection to this proposal is that it puts the cart before the horse. It is inadequate to say that a theory is successful because it survives. We should rather say that a theory survives because it is successful. The kinetic theory of heat makes true predictions and then survives. It is not the case that the theory 
survives and then makes true predictions. The success is temporally prior to the survival. Furthermore, there is a causal relationship between success and survival. Success is the cause, and survival is the effect. An effect is generally explained in terms of its cause, not vice versa. Thus, it is appropriate to say that the kinetic theory of heat survives because it makes true predictions, but inappropriate to say that the theory makes true predictions because it survives. In short, the explanans and the explanandum of the selectionist explanation should switch places.

Putnam's realist explanation does not have a conceptual flaw like the selectionist explanation, but it is plagued by a different sort of problem. Laudan (1981) claims that past theories, such as the Ptolemaic theory, the phlogiston theory of combustion, and the caloric theory of heat, were successful but not even approximately true. In response, however, Kitcher (1993: 140-149) and Psillos (1999, Chapter 5 and 6) distinguish between idle and working posits of a past theory, claiming that idle posits are false, but working posits are true in the present light, making the past theories approximately true. Thus, Laudan's criticism does not knock down realism.

Overall, the realist explanation is superior to the evolutionary explanation. The evolutionary explanation is inadequate due to the conceptual flaw pointed out above. Its explanatory scheme cannot be applied to the success of an individual theory. In contrast, the realist explanation does not have such a conceptual problem. It is applicable to the success of a particular theory. Consequently, we have prima facie reason for thinking that the realist explanation has a broader scope than the selectionist explanation.

A selectionist might object that selectionism is not intended to explain why a theory is successful, so the fact that selectionism cannot explain why a theory is successful is not a strike against it. How can you accuse a theory of not being able to explain phenomena it is not intended to explain? You cannot reject, for example, evolutionary theory on the grounds that it cannot explain why a solar eclipse occurs. It is the job of astronomical theory, not evolutionary theory, to explain why a solar eclipse occurs.

My response to the foregoing objection is to point out that it does not matter whether selectionism is intended to explain why a theory is successful or not. What matters is that realism can, but selectionism cannot, explain why a theory is successful. Consider that neither Ptolemy nor Copernicus intended to explain the phases of Venus, but Galileo's observation of the phases of Venus confirmed the Copernican theory and disconfirmed the Ptolemaic theory. From the Ptolemaic point of view, it is mysterious why Venus undergoes the phases. Similarly, from the selectionist point of view, it is utterly inexplicable why a theory is successful. The existence of such phenomena in science constitutes prima facie reason for thinking that realism is better than selectionism.

\section{Why do We Have Successful Theories?}

Leplin (1997: 9), Stanford (2000: 272), and Wray (2007: 83) claim that the explanandum of van Fraassen's evolutionary explanation is the existence of successful theories, so van Fraassen's suggestion is that successful theories exist today because successful theories survive and unsuccessful ones die:

van Fraassen thinks that we need to explain how it is that we have come to have successful theories. (Wray, 2007: 83)

It makes sense that we have successful theories because successful theories are selected and unsuccessful ones are eliminated. Thus, the evolutionary explanation is an attempt to illuminate the historical process which produced successful present theories. 
It is self-defeating for van Fraassen and Wray to use evolutionary theory to defend antirealism. They speak as if they believe that evolutionary theory is true. Let me quote just two sentences from Wray's writings:

Darwin tells us that the best explanation for the remarkable fit between organisms and the environments they inhabit is that natural selection ensures that organisms lacking such a fit are destroyed (see Darwin 1859/2003). (Wray, 2007: 84)

The selection mechanism operative in science, like natural selection in the biological world, is essentially an eliminative process, getting rid of the least fit alternatives. (Wray, 2010: 376)

Note that Wray's sentences indicate that he believes that evolutionary theory is true. It seems to me that if evolutionary theory is worthy of our belief, so are all other scientific theories, such as the kinetic theory of heat and the special theory of relativity, given that there is no relevant difference among these theories. Thus, the antirealist use of evolutionary theory opens a door to realism. The selectionist explanation, however, is intended to be an antirealist alternative to the realist explanation.

Wray (2010) rejects the realist explanation, citing Stanford's new pessimistic induction (2006) viz., successful present theories will be replaced with rival theories hitherto unconceived because successful past theories were superseded by the then unconceived rival theories:

Consequently, it seems likely that future developments in a field will reveal additional competing theories that are also able to account for the data, theories, though, that are now unconceived alternatives. (Wray, 2010: 371)

Stanford's new pessimistic induction clashes with the realist suggestion that we have successful theories because they are (approximately) true. The realist explanation seems to be doomed.

It is problematic, however, for Wray to embrace the evolutionary explanation and to invoke Stanford's new pessimistic induction because the pessimistic induction implies that successful theories, including evolutionary theory, are false. He cannot talk as if evolutionary theory is true while wielding the pessimistic induction against realism. Wray might now say that the selectionist explanation is empirically adequate. Let me point out, however, that the pessimistic induction also implies that successful theories, including evolutionary theory, are empirically inadequate:

The successful past theories turned out to be empirically inadequate. So successful current theories will turn out to be empirically inadequate as well. (Park, 2001: 78)

Most of these theories eventually turned out not to be empirically adequate. Therefore, we should believe that probably, most of the theories we currently accept are not empirically adequate either. (Lange, 2002: 282)

In a nutshell, the pessimistic induction entails that evolutionary theory, although successful, is neither true nor empirically adequate. Therefore, Wray cannot embrace both the selectionist explanation and the pessimistic induction at the same time.

To meet this challenge, the selectionist might appeal to the realist response to the pessimistic induction in the literature. Recall that Kitcher (1993: 140-149) and Psillos (1999, Chapter 5 and 6) argue that successful past theories are approximately true because some of 
their components are true. Relying on their insight, the selectionist might argue that his evolutionary explanation is approximately true.

Wray, however, cannot avail himself of the realist response to the pessimistic induction because this approach is tantamount to the rejection of antirealism and acceptance of realism. The realist response asserts that successful current scientific theories, such as the kinetic theory of heat and oxygen theory of combustions, are approximately true, which is exactly what realism affirms and antirealism denies.

Wray might now reply that he did not claim or believe that evolutionary theory is true when he offered the selectionist explanation. His aim was to refute the realist explanation, and he achieved his aim by proposing the selectionist alternative to it. Since he did not claim that evolutionary theory is true in the first place, his position is not refuted by the pessimistic induction. He can only enjoy watching the realist explanation destroyed by the pessimistic induction.

Let me point out, however, that many sentences in Wray's papers $(2007,2010)$ clearly show that he believed that the evolutionary explanation is true. Two such sentences are cited earlier in this section. In addition to those sentences, consider the following sentence which expresses the core idea of selectionism:

Selectionist: Successful theories exist today because successful theories survive and unsuccessful ones die.

Note that by giving this explanation, the selectionist is claiming and expressing his belief that successful theories exist today because successful theories survive and unsuccessful ones die. How can he say, "Successful theories exist today because successful theories survive and unsuccessful ones die" without claiming or believing that successful theories exist today because successful theories survive and unsuccessful ones die?

Wray would now retort that there was a gap between his belief and his language when he defended the selectionist explanation. He did not believe that successful theories exist today because successful theories survive and unsuccessful ones die, although he said, "Successful theories exist today because successful theories survive and unsuccessful ones die." His previous sentences should be construed not literally but non-literally. Thus, none of his beliefs is refuted by the pessimistic induction.

Let me point out, however, that your opponent can escape any criticism by saying "There was a gap between my belief and my language." In other words, you can refute no position, how absurd it might be, if your opponent plays the gap card. Consider the following imaginary dialogue between you and your opponent:

Opponent: The earth is flat. After all, the horizon looks flat.

You: Look at this picture of the earth taken from the satellite. It's round.

Opponent: Oh, I believed all along the earth is round! My position is not refuted by your picture. My previous sentence 'The earth is flat' should be interpreted not literally but non-literally.

Note that your opponent plays the gap card solely for the sake of diverting your criticism. His use of the gap card is arbitrary. He needs to provide criteria to distinguish between the contexts in which his sentences should be construed literally and non-literally. The same criticism applies to the selectionist. It is arbitrary for the selectionist to play the gap card solely for the sake of diverting the criticism that the pessimistic induction refutes the 
selectionist explanation. He needs to provide criteria to distinguish between the cases in which his sentences should be interpreted literally and non-literally.

\section{Superiority}

It should be clear now that an antirealist has every reason to reject the evolutionary explanation. Interestingly, Wray (2007: 85-88, 2010: 375-376) argues that the evolutionary explanation is superior to the realist explanation. Let me focus on his central assertion that there are two phenomena in science that the selectionist can explain, but the realist cannot. Those phenomena are failures and competitions of scientific theories. Let me explore these phenomena one by one.

There were failures of scientific theories throughout the history of science, viz., some successful theories have died, including the Ptolemaic theory, the phlogiston theory of combustion, and the caloric theory of heat. Why did these theories die? Wray answers that they died because they ran into anomalies. For example, Galileo observed the phases of Venus and the moons of Jupiter with his telescope, and the Ptolemaic theory could not explain these celestial phenomena. As a result, the Ptolemaic theory "came to be regarded as unacceptable" (Wray, 2007: 87).

In my view, Wray's evolutionary account of the failures of scientific theories can be cheerfully endorsed by the realist. After all, the collision of successful past theories with anomalies is compatible with the realist contention that successful theories are approximately true. An approximately true theory does not perfectly describe unobservables, so it may be empirically inadequate and allows anomalies. No realist would claim that an approximately true theory is necessarily empirically adequate, and that past theories are empirically adequate. Wray is aware that the concept of approximate truth exists in the realist toolbox:

No doubt realists will claim that they can reconcile the rejection of past successful theories with their claim that our current predictively accurate theories are apt to be true, or approximately true. (Wray, 2007: 86)

Surprisingly, however, Wray does not explore how a realist can use the concept of approximate truth to confront the historical challenge that successful theories clashed with anomalies.

Let me now turn to Wray's criticism that the realist cannot explain the failures of past theories. On what ground does he think so?

The realist seems to have nothing to say here. The realist cannot say in good conscience that a theory that once reflected the structure of the world does not do so any longer. (Wray, 2007: 86)

The realist cannot say that the Ptolemaic theory, for example, died because it was once true. After all, the truth-value of a theory does not change, although the amount of evidence for or against it changes.

No realist would say, however, that past theories died because they no longer accurately reflected the structure of the world. A realist would rather say that past theories died because their competitors were closer to the truths. For example, the Ptolemaic theory died because its competitor, the Copernican theory, was more successful and hence closer to the truth. Thus, realism can explain the failures of past theories. Wray, however, anticipates this suggestion and critically responds to it as follows:

..the realist claims that once successful theories are now rejected because they have been superseded by theories that better represent the unobservable structure of the world. But, even 
this explanatory strategy is suspect. After all, as both Laudan (1984) and Carrier (1991) note, the predictive success of some of our theories is a consequence of something other than the fact that these theories accurately represent the world. (Wray, 2007: 86)

Given that there is no connection between success and truth, as the history of science suggests, there is no connection either between greater success and being closer to truth. Therefore, the realist cannot say that the Ptolemaic theory died because the Copernican theory was more successful and hence closer to the truth than the Ptolemaic theory.

We can grant that all the successful past theories are false, so success is not a reliable indicator of truth. It does not follow, however, that more success is not a reliable indicator of being closer to truth. To use an analogy, suppose that one hundred people are located in Los Angeles. All of them take a step toward the White House, but none of them arrives in the White House. Thus, taking a step toward the White Hours is not a reliable indicator of being in the White House. Even so, taking a step toward the White House is a reliable indicator of being closer to the White House. Similarly, none of the successful past theories is true, so success is not a reliable indicator of truth. This historical fact, however, does not preclude the possibility that the past theories are all closer to the truths than their predecessors. In other words, even if they did not arrive at truths, they might be closer to the truths than their precursors.

Let me now reduce to absurdity Wray's contention that more success is not a reliable indicator of being closer to truth. The Ptolemaic theory contains unbelievable claims that the earth is stationary at the center of the universe, that the celestial sphere turns around the earth once a day, that the sun is a planet, and so forth. In contrast, the contemporary Copernican theory claims that the earth moves around the sun, that there is no such thing as the celestial sphere, that the sun is a star, and so on. The corollary of Wray's contention is that the contemporary Copernican theory is not closer to the truth than the Ptolemaic theory. The corollary strikes me as absurd.

Let me turn to Wray's contention that the selectionist can, but the realist cannot, explain competitions among scientific theories. The Ptolemaic theory competed with the Copernican theory in the late $16^{\text {th }}$ century (Wray, 2007: 87). Why did the two theories coexist in the past? A selectionist answers as follows:

When two competing theories both enable scientists to make accurate predictions of observable phenomena, we should expect each theory to be accepted by some scientists. (Wray, 2007: 88).

The Ptolemaic theory and the Copernican theory coexisted in the $16^{\text {th }}$ century because they equally made accurate predictions. Their equal capacity to make true predictions enabled them to coexist. From the selectionist point of view, when two theories have the equal predictive power, none of them is eliminated, i.e., both are selected.

In contrast, Wray claims, realism cannot explain the competitions of scientific theories. The realist cannot say that the Ptolemaic theory and the Copernican theory coexisted because the two "theories accurately represent the underlying structure of the world" (Wray, 2007: 87). After all, the Ptolemaic theory does not accurately represent the world. Neither does the Copernican theory. The Copernican theory is false because it makes the false claim that the sun is at the center of the universe. The competition of the two theories is an anomaly to realism.

Wray's criticism against realism is unfair. No realist would say that the Ptolemaic theory and the Copernican theory coexisted because these theories accurately represent the underlying structure of the world. A realist would rather say that they coexisted because both theories approximately represent the underlying structure of the world, and neither theory 
was revealed to be closer to the truth than the other. Again, Wray does not explore how the realist can use the concept of approximate truth to diffuse his criticism against realism.

Suppose that Wray is right that selectionism can explain the failures and the competitions of scientific theories, but realism cannot. What does this scenario prove? This scenario proves that selectionism is more successful than realism, but Wray cannot say that selectionism is closer to the truth than realism because he previously rejected the realist idea that more success is a reliable indicator of being closer to truth. In short, his criticism of realism can be turned against his own position.

Wray claims that the selectionist explanation has a virtue over the realist explanation because his explanation complies with the symmetry thesis of the Strong Programme in the sociology of scientific knowledge:

The fact that van Fraassen's selectionist explanation enables us to explain both the success and failures of science should appease proponents of the Strong Programme in the sociology of scientific knowledge. Their symmetry principle demands that the same causes explain both the success and failures of scientists (see Barnes and Bloor 1982, 22-23). (Wray, 2007: 87 footnote)

The selection process explains why we accept successful theories and reject unsuccessful theories, which echoes the symmetry thesis of the Strong Programme stating that the "same types of cause would explain, say, true beliefs and false beliefs" (Bloor, 1991: 7).

Two comments are in order. First, realism can explain both the success and failures of science, pace Wray. Second, selectionism does not meet the demand of the reflexivity thesis, another tenet of the Strong Programme, stating that "its patterns of explanations must be applicable to sociology itself" (Bloor, 1991: 7). Bloor advances the reflexivity thesis for fear that if sociology does not abide by the reflexivity thesis, "sociology would be a standing refutation of its own theories" (Bloor, 1991: 7). Bloor does not want the Strong Programme to be self-defeating. Thus, the Strong Programme has a theoretical mechanism ensuring that it is free of contradiction. Scientific antirealism, in contrast, lacks such a mechanism. Recall that the pessimistic induction backfires on the selectionist explanation.

\section{Approximate Truth}

My defense of realism in this paper relies on the notion of approximate truth. An antirealist might object that the concept is vague, and that no precise definition of it is forthcoming. I admit that this objection is legitimate. It requires, however, separate papers to fully defend the realist predicate. I can only weaken the objection here with the following two considerations. First, the antirealist needs the vague predicate too. Suppose that an experiment was performed correctly, but there is a small gap between an actual outcome and an expected outcome. In such a case, the relevant observational consequence of a theory is approximately true (Park, forthcoming). If, however, the antirealist dismisses the predicate for being vague, he can only say that the observational consequence is false.

Second, a realist can vindicate the vague predicate 'approximate truth' in the way that van Fraassen vindicates the vague predicate 'observable.' In response to the objection that the antirealist predicate 'observable' is vague, van Fraassen claims that the vague predicate is "usable provided it has clear cases and clear counter-cases" (1980: 16). Some objects, such as cats and chairs, are clearly observable. Some objects, such as electrons and neutrinos, are clearly unobservable. Other objects are in between. Similarly, I claim, the vague predicate 'approximate truth' is viable, insofar as there are clear cases and counter-cases of approximately true description. If it is exactly true that Bill Clinton is $180.2 \mathrm{~cm}$, it is 
approximately true that he is $180 \mathrm{~cm}$, and it is completely false that he is $18 \mathrm{~cm}$. In sum, 'approximate truth' and 'observable' are on the same boat.

\section{Conclusion}

A selectionist and a realist have different explanations for the existence of successful theories. The selectionist argues that we have successful theories because successful theories survive and unsuccessful ones die. In contrast, the realist argues that we have successful theories because they are approximately true. Wray conjures up the pessimistic induction to demonstrate the superiority of the selectionist explanation over the realist explanation.

It is problematic for Wray to embrace both the selectionist explanation and the pessimistic induction because the pessimistic induction entails that evolutionary theory is neither true nor empirically adequate. He might reply that he does not believe that evolutionary theory is true, although he uses it to explain the existence of successful theories. Thus, the pessimistic induction does not refute his position. I replied that you can refute no position, however preposterous it might be, if your opponent plays the gap card, and that it is arbitrary for the selectionist to play the gap card solely for the sake of diverting the criticism that the pessimistic induction refutes the selectionist explanation.

Wray argues that the evolutionary explanation is better than the realist explanation because the evolutionary explanation can accommodate the failures and the competitions of scientific theories, whereas the realist explanation cannot. I replied that on close examination, the realist explanation can cope with both the failures and the competitions of scientific theories. Wray did not explore how a realist can utilize the concept of approximate truth to diffuse his objections against realism.

Finally, selectionism lacks a theoretical resource to explain certain phenomena in science, but realism does not. Recall that the selectionist cannot explain why a particular theory is successful due to the conceptual problem, viz., it is inadequate to assert that a theory is successful because it survived. In contrast, there is no conceptual flaw with the realist suggestion that a theory is successful because it is approximately true. Current analysis indicates that realism is preferable to selectionism, contrary to what Wray claims.

\section{References}

Barnes, Barry and David Bloor (1982). "Relativism, Rationalism and the Sociology of Knowledge. In M. Hollis \& S. Lukes (eds.), Rationality and Relativism. Cambridge: MIT Press: 21-47.

Bloor, David (1991). Knowledge and Social Imagery. Chicago and London: The University of Chicago Press.

Carrier, Martin (1991). "What is Wrong with the Miracle Argument?" Studies in History and Philosophy of Science 22(1): 23-36.

Darwin, Charles (1859/2003). On the Origin of Species by Means of Natural Selection. edited by J. Carroll. Peterborough: Broadview Press, Ltd.

Kitcher, Philip (1993). The Advancement of Science: Science without Legend Objectivity without Illusion. New York: Oxford University Press. 
Lange, Marc (2002). "Baseball, Pessimistic Inductions and the Turnover Fallacy", Analysis 62 (4): $2881-85$.

Laudan, Larry (1981). “A Confutation of Convergent Realism”, Philosophy of Science 48(1): $19-49$.

(1984). "Explaining the Success of Science: Beyond Epistemic Realism and Relativism. In J. T. Cushing, C. F. Delaney, \& G. Gutting (eds.), Science and Reality: Recent Work in the Philosophy of Science. Notre Dame: University of Notre Dame: 83-105.

Leplin, Jarrett (1997). A Novel Defense of Scientific Realism. New York: Oxford University Press.

Park, Seungbae (2001). Scientific Realism vs. Scientific Antirealism. Ph.D. Dissertation. University of Arizona.

-------- (forthcoming). “Approximate Truth vs. Empirical Adequacy”, Epistemologia.

Psillos, Stathis (1999). Scientific Realism: How Science Tracks Truth. New York: Routledge.

Putnam, Hilary (1975). Mathematics, Matter and Method (Philosophical Papers, vo. 1), Cambridge: Cambridge University Press.

Stanford, Kyle (2000). "An Antirealist Explanation of the Success of Science”, Philosophy of Science 67(2): 266-284.

(2006). Exceeding Our Grasp: Science, History, and the Problem of Unconceived Alternatives. Oxford: Oxford University Press.

van Fraassen, Bas (1980). The Scientific Image. Oxford: Oxford University Press.

Wray, K. Brad (2007). "A Selectionist Explanation for the Success and Failures of Science", Erkenntnis 67 (1): 81-89.

(2010). “Selection and Predictive Success”, Erkenntnis 72 (3): 365-377. 